\title{
A Novel Non-Line-of-Sight Indoor Localization Method for Wireless Sensor Networks
}

\author{
Yan Wang $\mathbb{D}^{\mathbb{B}}$, Xuehan $\mathrm{Wu}$, and Long Cheng $(\mathbb{D}$ \\ Department of Computer and Communication Engineering, Northeastern University, Qinhuangdao 066004, China \\ Correspondence should be addressed to Yan Wang; ywang8510@gmail.com and Long Cheng; chenglong8501@gmail.com
}

Received 12 April 2018; Accepted 5 August 2018; Published 27 September 2018

Academic Editor: Grigore Stamatescu

Copyright (C) 2018 Yan Wang et al. This is an open access article distributed under the Creative Commons Attribution License, which permits unrestricted use, distribution, and reproduction in any medium, provided the original work is properly cited.

\begin{abstract}
The localization technology is the essential requirement of constructing a smart building and smart city. It is one of the most important technologies for wireless sensor networks (WSNs). However, when WSNs are deployed in harsh indoor environments, obstacles can result in non-line-of-sight (NLOS) propagation. In addition, NLOS propagation can seriously reduce localization accuracy. In this paper, we propose a NLOS localization method based on residual analysis to reduce the influence of NLOS error. The time of arrival (TOA) measurement model is used to estimate the distance. Then, the NLOS measurement is identified through the residual analysis method. Finally, this paper uses the LOS measurements to establish the localization objective function and proposes the particle swarm optimization with a constriction factor (PSOC) method to compute the position of an unknown node. Simulation results show that the proposed method not only effectively identifies the LOS/NLOS propagation condition but also reduces the influence of NLOS error.
\end{abstract}

\section{Introduction}

The rapid development of microelectromechanical system (MEMS) technology, sensor technology, wireless communication, and low-power embedded technology promotes the progress and development of wireless sensor networks (WSNs). WSNs consist of a large number of inexpensive microsensor nodes deployed in a monitored region. The sensor nodes are connected to each other by selforganization and multihop communications [1]. Sensor nodes consist of sensors, digital processing units, a wireless communication module, and a power module. They can collaboratively sense, gather, and process the information of the perceived objects in a monitored region and then send the information to the sink node. WSNs are widely used in traffic management, environmental monitoring, medical care networks, logistics management, and other fields and profoundly influence the social life of people [2].
One of the most important issues for WSNs is localization technology [3]. The localization technology is the essential requirement of constructing a smart building and smart city. WSN-based localization methods can be categorized as range-based localization methods and range-free localization methods. In range-based localization methods, different measurement techniques for localization can be classified as time of arrival (TOA), time difference on arrival (TDOA), received signal strength (RSS), and angle of arrival (AOA). The range-free localization methods do not need to measure the distance or angle between the nodes [4]. These methods can estimate position based on the network connectivity and the distribution of the history measurements. The range-free localization methods can be divided into multihop estimation-based localization and pattern matching-based localization.

For the TOA-based localization method, the signal velocity is known in advance. It measures the travel time of the signal from the beacon node to the unknown node, and the 
distance between two nodes is equal to the product of the signal velocity and the travel time. However, this method requires high-precision time synchronization between two nodes. As light synchronization error can significantly affect the ranging error. Therefore, the TOA method requires additional hardware to ensure the time synchronization. The TDOA method requires two different transceivers on a node so that the node can transmit two signals with different velocities at the same time. It estimates the distance by measuring the two signals' arrival time difference between the beacon node and the unknown node. The requirement of time accuracy of the TDOA method is lower than the TOA method, but it still has high requirements for hardware. The RSS method is one of the least expensive ways to locate an unknown node because it does not need additional hardware. The RSS method measures the signal power loss value from a beacon node to an unknown node, and it converts the power loss value to the distance through a signal propagation model. The AOA method measures and calculates the angles between beacon nodes and an unknown node and then estimates the position of the unknown node based on the angle between two nodes.

In this paper, we investigate the TOA-based localization method in an indoor environment. Obstacles can result in NLOS propagation in harsh indoor environments, and the accuracy of localization will drop sharply. We first propose an NLOS identification method based on residual analysis. The propagation condition can be identified by it. Then, the localization objective function is established using the LOS measurements. In addition, the particle swarm optimization with a constriction factor method is proposed to find the optimal solution of the localization function. The optimal solution is the estimated position of the unknown node. The main contributions of this paper are given as follows:

(1) The NLOS identification method does not need prior knowledge of the NLOS error. In addition, it can identify the NLOS measurements when the number of LOS measurements is larger than the number of NLOS measurements.

(2) The proposed NLOS correction method can mitigate the effect of the NLOS error.

(3) The proposed method not only uses TOA measurements but also uses other signal features such as TDOA and RSS easily. Therefore, it is not constrained by different physical measurement techniques.

The rest of the paper is organized as follows. Section 2 analyzes the NLOS localization technology for WSNs. Section 3 introduces the proposed NLOS identification methods based on residual analysis and a localization method based on an intelligent optimization algorithm. In Section 4, the simulation results of the proposed algorithm are presented, and the performance of the proposed algorithm is analyzed. The conclusions are presented in Section 5.

\section{Related Work}

Compared with traditional positioning systems, WSNbased localization systems can be quickly deployed and can adapt to various harsh environmental conditions. They have the characteristics of low power consumption, low cost, and strong expansibility. In addition, the Global Positioning System (GPS) technique, which is widely used at present, has the characteristics of high energy consumption, high cost, and large volume compared with WSNs [5]. Thus, WSN-based localization systems have broad application prospects, and they can be used in environmental monitoring, medical care networks, military applications, target tracking, intelligent traffic management, and other fields. The development of WSN-based localization technology has promoted an industrial revolution that influences the social life of people.

Because the WSN localization technology has remarkable superiority, both researchers and designers are paying more attention to it and devoting more effort to improving the positioning accuracy. In [6], a residual test method is proposed to determine the number of LOS and identify the propagation condition synchronously. This method can identify the NLOS with high accuracy. In [7], the authors proposed a routing algorithm that is widely used in centralized range-based localization schemes. Experimental results show that the algorithm provides distance estimates with low estimation error. However, the algorithm requires a large amount of calculation. A novel localization algorithm based on an approximate convex decomposition (ACDL) is proposed [8]. It relies only on network connectivity information. The hop count distance between nodes can provide a good approximation of the Euclidean distance. In [9], the authors design a localization method with outlier detection, and the ranges with large errors can be eliminated explicitly before computing the location. However, the method must define verifiable graphs in which all edges should be verifiable. To obtain a low complexity, the authors proposed a modification of the gradient descent method and an accurate multilateration localization algorithm for wireless sensor networks [10]. Only when using the RSSI to estimate the distances between nodes, the proposed algorithm can obtain better convergence properties and a lower computational load in the presence of significant range error.

NLOS propagation is ubiquitous in practical indoor environments. NLOS propagation will contribute a positive additional excessive delay to the measured value. NLOS error is the main source of the localization error. To improve the positioning accuracy in practical conditions, NLOS identification and mitigation methods are widely investigated. A residual weighting algorithm (Rwgh) is proposed in [11]. The sum of squared residuals of a least squares estimation is used as the indicator to show the accuracy of the calculated node coordinates. Least squares multipoint location is applied on all possible combinations of the distance measurements. Then, the authors compute the estimated location and used it as a weighted combination of these intermediate estimates. The RANSAC 
algorithm is an iterative method to estimate the position from a set of measurements that contains NLOS error [12]. A reasonable result is produced only with a certain probability, so RANSAC is a nondeterministic algorithm in this sense. The probability can be increased as more iterations are allowed. In [13], the authors proposed a distributed multiple-model estimator for simultaneous localization and tracking (SLAT) with NLOS mitigation. The difficulties of exponentially growing terms for centralized multiple-model estimation can be overcome if the fusion is carried out in a distributed manner. An NLOS mitigation technique based on convex SDP optimization is proposed in [14]. Especially in severe NLOS environments, the proposed SDP estimator outperforms the other algorithms substantially. In [15], a novel algorithm is presented by the authors to solve NLOS propagation. The algorithm depends only on the features extracted from the received waveform. In addition, there is no need to formulate an explicit statistical model for the features.

\section{System and Range Measurement Model Description}

In this section, we first analyze the TOA measurement model in LOS and NLOS propagation conditions, respectively. Then, we propose an NLOS identification method based on residual analysis, according to the characteristics of the NLOS error. Finally, we improve the existing NLOS localization method by using particle swarm optimization with a constriction factor.

3.1. TOA Measurement Model. The TOA method measures the travel time of a signal from the beacon node to the unknown node. The true distance of TOA is modeled as follows:

$$
d=c \cdot t
$$

where $c$ is the speed of the signal, $d$ is the distance between the two nodes, and $t$ is the travel time of the signal between the two nodes.

Because the travel time $t$ cannot be completely synchronous in LOS propagation conditions, it consists of measurement error. The time estimation of TOA is as follows [16]:

$$
\widehat{t}=t+n_{i t}
$$

where $t$ is the true travel time of the signal between the two nodes; $n_{i t}$ is the measurement error modeled as a zero-mean white Gaussian process with variance $\sigma_{i t}^{2}$. The distance between the $i$ th beacon node and the unknown node in LOS propagation conditions is as follows [17]:

$$
\widehat{d}_{i}=c \cdot\left(t+n_{i}\right)=c \cdot t_{i}+n_{i}=d_{i}+n_{i}
$$

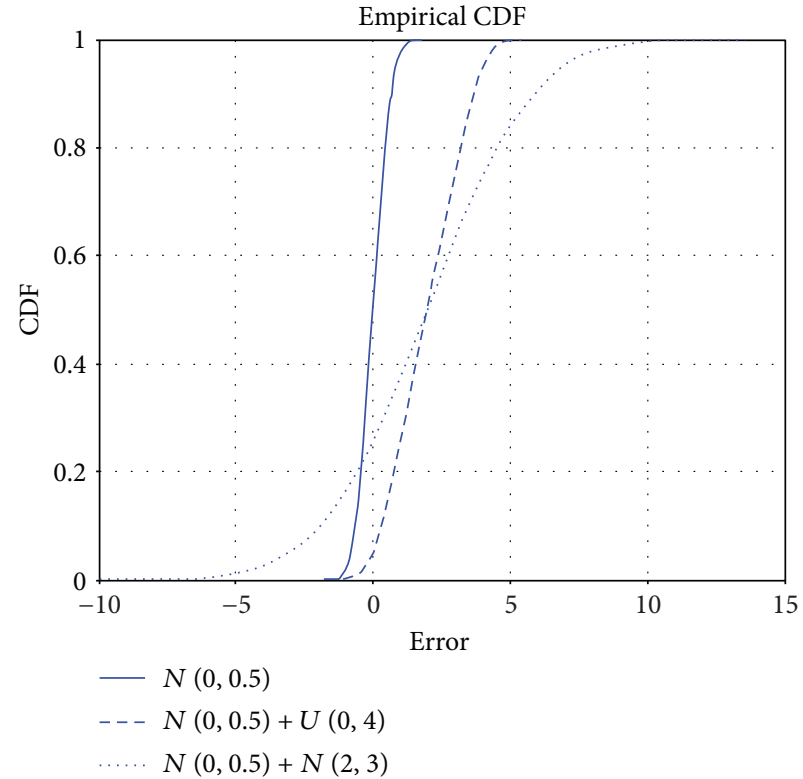

Figure 1: The CDF for measurement noise and NLOS error.

where $d_{i}$ is the true distance between the two nodes; $n_{i}$ is the measurement error modeled as a zero-mean white Gaussian process with variance $\sigma_{i}^{2}$.

In practical conditions, the existence of obstacles will result in NLOS conditions. Such obstacles will admit a positive error component to the estimated distance. Considering the NLOS error, the distance between the $i$ th beacon node and the unknown node in NLOS propagation conditions is modeled as follows [18, 19]:

$$
\widehat{d}_{i}=d_{i}+n_{i}+n_{\mathrm{NLOS}}
$$

where $n_{\mathrm{NLOS}}$ is the NLOS error and it is the positive bias error, and $n_{\mathrm{NLOS}}$ is uniformly distributed $\left(n_{\mathrm{NLOS}} \sim U\left(0, B_{\max }\right)\right)$. Because the causes of NLOS error and measuring error are different, NLOS error is assumed to be independent of the measuring error [20].

Figure 1 shows the cumulative distribution function (CDF) of measurement noise and NLOS error. The measurement noise $n_{i}$ obeys a Gaussian distribution, that is, $n_{i} \sim$ $N(0,0.5)$. The NLOS error is a uniform distribution or a Gaussian distribution, that is, $n_{\mathrm{NLOS}} \sim U(0,4)$ and $n_{\mathrm{NLOS}} \sim$ $N(2,3)$.

3.2. An NLOS Identification Method Based on Residual Analysis. NLOS propagation is ubiquitous in practical conditions and has a large influence on measurements. To obtain more accurate measurements, approaches to reduce the influence that NLOS error admits to localization accuracy must be considered. NLOS error has distinct characteristics compared with the measuring error: (1) NLOS error is always positive. (2) The standard deviation of the distance measurement in NLOS propagation conditions is 
larger than that in LOS propagation conditions. (3) NLOS error exhibits high randomness.

Considering the characteristics of the NLOS error, NLOS identification methods based on residual analysis can be used to determine and eliminate the NLOS measurements. The basic approach of the residual analysis method can be expressed as follows:

Step 1. There are $N$ different beacon nodes in the field. Combine the measurements provided by these beacon nodes. $M$ different combinations of distance measurements can be obtained.

$$
M=\sum_{i=3}^{N} C_{N}^{i}
$$

Step 2. Use the maximum likelihood method to compute the estimated location of each combination. The estimated position of the $k$ th combination is $\widehat{X}_{k}$. The details of the maximum likelihood method are shown in Appendix. Calculate the residual of each measurement as follows:

$$
\operatorname{Re} s_{i}(k)=\left|\widehat{d}_{i}-\left\|\widehat{X}_{k}-X_{i}\right\|\right| \text {, }
$$

where $\hat{d}_{i}$ is the distance from the unknown node to the $i$ th beacon node, and $X_{i}$ is the coordinate of the $i$ th beacon node.

Step 3. Accumulate the residuals of each measurement as follows:

$$
\mathrm{CRes}_{i}=\sum_{k=1}^{M} \operatorname{Res}_{i}(k)
$$

We can obtain $N$ cumulative residuals CRes $_{i}, \quad i=1$, $\ldots, N$.

Step 4. Sort the cumulative residuals from large to small; the measurements with the largest residual can be regarded as NLOS measurements.

By using the above steps, we can determine the NLOS measurements, and the rest of the measurements can be regarded as measurements in LOS propagation conditions.

3.3. PSO with a Constriction Factor-Based Localization Method. The probability density function of the measurement in an LOS condition can be expressed as follows [21]:

$$
f_{\mathrm{LOS}}\left(d_{i}\right)=\frac{1}{\sqrt{2 \pi \sigma_{i}^{2}}} \exp \left(-\frac{\left(\hat{d}_{i}-d_{i}\right)^{2}}{2 \sigma_{i}^{2}}\right),
$$

where $\widehat{d}_{i}$ is the measured distance from a beacon node to an unknown node, $d_{i}=\sqrt{\left(x_{i}-x\right)^{2}+\left(y_{i}-y\right)^{2}}$ is the true distance between the $i$ th beacon node and an unknown node, $\left(x_{i}, y_{i}\right)$ is the coordinate of the $i$ th beacon node, and $(x, y)$ is the true location of the unknown node. We use the LOS measurements to establish the objective function as follows:

$$
(\widehat{x}, \widehat{y})=\arg \max \left\{\prod_{i=1}^{L} f_{\mathrm{LOS}}\left(d_{i}\right)\right\},
$$

where $L$ is the number of LOS measurements.

To solve the position function directly, not only is a large amount of calculation required but the difficulty of finding an analytical solution is also encountered. Therefore, we use the particle swarm optimization with a constriction factor (PSO-C) method to determine the optimal solution. PSO is based on simulating a simplified model of social interaction. PSO is easy to implement and does not require gradient information, so it is widely used in scientific research and engineering practice.

The basic principle of the algorithm is as follows: assume that a swarm includes $M$ particles. The search space is a $D$-dimensional vector. The location of the $i$ th particle in the swarm is $X_{i}=\left(x_{i 1}, x_{i 2}, \ldots, x_{i D}\right)$. The velocity is $v_{i}=\left(v_{i 1}\right.$, $\left.v_{i 2}, \ldots, v_{i D}\right)$. The experienced best location of a particle is $p_{i}=\left(p_{i 1}, p_{i 2}, \ldots, p_{i D}\right)$, where $1 \leq i \leq m$. The experienced best location of all particles in the swarm is $p_{g}=\left(p_{g 1}, p_{g 2}, \ldots\right.$, $\left.p_{g D}\right)$. The location and velocity of the particles change according to the equation as follows:

$$
v_{i D}^{k+1}=K\left[v_{i D}^{k}+c_{1} \xi\left(p_{i D}^{k}-x_{i D}^{k}\right)+c_{2} \eta\left(p_{g D}^{k}-x_{i D}^{k}\right)\right],
$$

$$
x_{i D}^{k+1}=x_{i D}^{k}+v_{i D}^{k+1}
$$

$K$ is a constriction factor and is a function of $c_{1}$ and $c_{2}$.

$$
K=\frac{2}{\left|2-\varphi-\sqrt{\varphi^{2}-4 \varphi}\right|}, \quad \varphi=c_{1}+c_{2}>4,
$$

where $c_{1}$ and $c_{2}$ are learning factors, and where $c_{1}=c_{2}=2.05$. $\xi$ and $\eta$ are two uniform random numbers in $[0,1]$, that is, $\xi, \eta \in U(0,1)$. The velocity of the particles is limited to a maximum range $V_{\max }$. $V_{\max }$ determines the search ability of particles in the search space.

The pseudocode of the PSO-C strategy is shown as follows: 


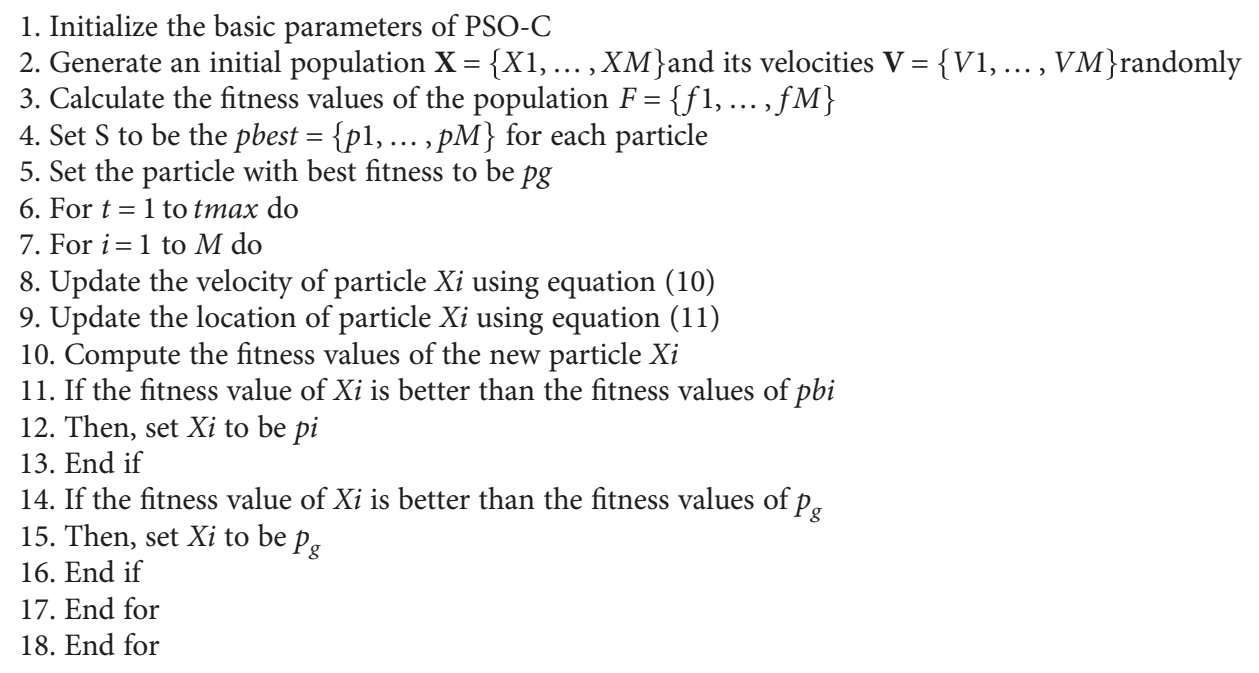

Pseudocode 1

The $p_{g}$ is the estimated position of an unknown node.

\section{Simulation and Experiments Results}

In this section, we evaluate the performance of our proposed NLOS localization algorithms. We compare the proposed method with RANSAC [12], ML [22], and Rwgh [11] methods. The $N$ beacon nodes and one unknown node are randomly deployed in a $30 \mathrm{~m} \times 30 \mathrm{~m}$ square space. One obstacle is randomly deployed in the field. The communication range of sensor node is $50 \mathrm{~m}$. The measurement error $n_{i}$ is modeled as a zero-mean white Gaussian process with variance $\sigma_{i}^{2}$. The NLOS error $n_{\text {NLOS }}$ obeys the uniform distribution $\left(n_{\mathrm{NLOS}} \sim U\left(0, B_{\max }\right)\right)$. The simulation results are obtained through 1000 Monte Carlo runs. The default parameter values in the simulation are shown in Table 1. We consider the average localization error (ALE) as the performance metric.

$$
\mathrm{ALE}=\frac{1}{M} \sum_{i=1}^{M} \sqrt{(\widehat{x}(i)-x(i))^{2}+(\widehat{y}(i)-y(i))^{2}}
$$

where $M=1000,[x(i), y(i)]$ is the true location of the mobile node, and $[\widehat{x}(i), \hat{y}(i)]$ is the estimated location for the ith Monte Carlo run.

First, the identification success rate of the proposed method is evaluated. Figure 2 shows the identification success rate versus the number of beacon nodes. In this simulation, the standard variance of the measurement noise in the LOS condition $\sigma_{i}$ is varied from 0.1 to 0.5 , and the number of beacon nodes is varied from 5 to 10 . The results show that as the number of beacon nodes increases, the success rate of the proposed method increases. In addition, as the value of $\sigma_{i}$ increases, the success rate of the proposed method decreases because, as the value of $\sigma_{i}$ increases, the measurements will be disturbed by measurement noise more seriously.
TABLE 1: The default parameter values.

\begin{tabular}{lcc}
\hline Parameters & Symbol & Default values \\
\hline $\begin{array}{l}\text { Number of beacon nodes } \\
\text { The standard deviation of } \\
\text { the measurement noise }\end{array}$ & $N$ & 8 \\
The NLOS errors & $N\left(\mu_{\mathrm{NLOS}}, \sigma_{\mathrm{NLOS}}^{2}\right)$ & $\mu_{\mathrm{NLOS}}=2, \sigma_{\mathrm{NLOS}}=7$ \\
The NLOS errors & $U\left(0, B_{\max }\right)$ & $B_{\max }=7$ \\
\hline
\end{tabular}

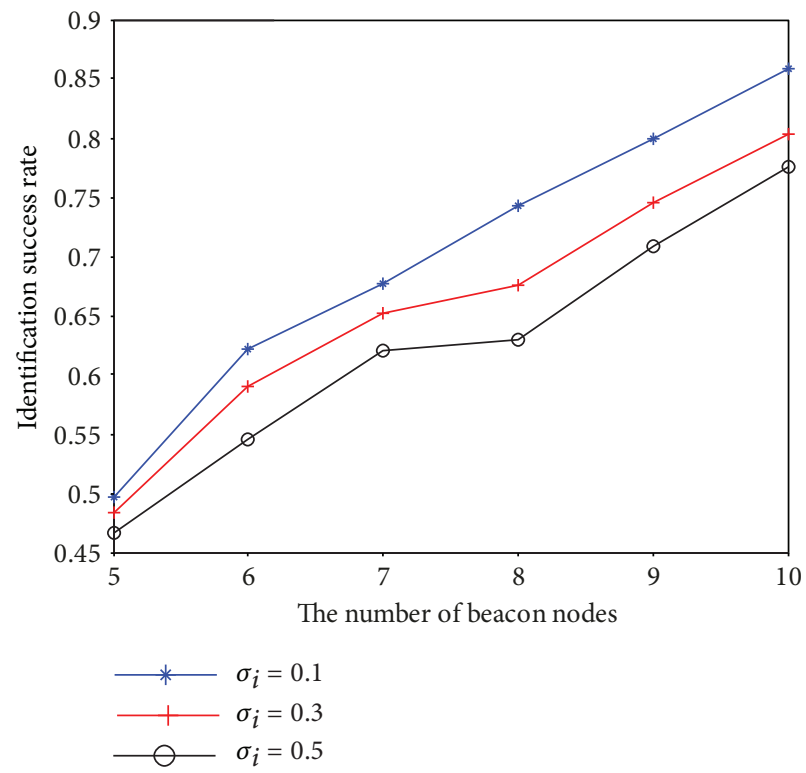

Figure 2: The identification success rate versus the number of beacon nodes.

When the NLOS error obeys the uniform distribution $\left(n_{\mathrm{NLOS}} \sim U\left(0, B_{\max }\right)\right)$, the identification success rate versus the maximum bias of NLOS error $B_{\max }$ is determined as shown in Figure 3. In this simulation, the standard variance 


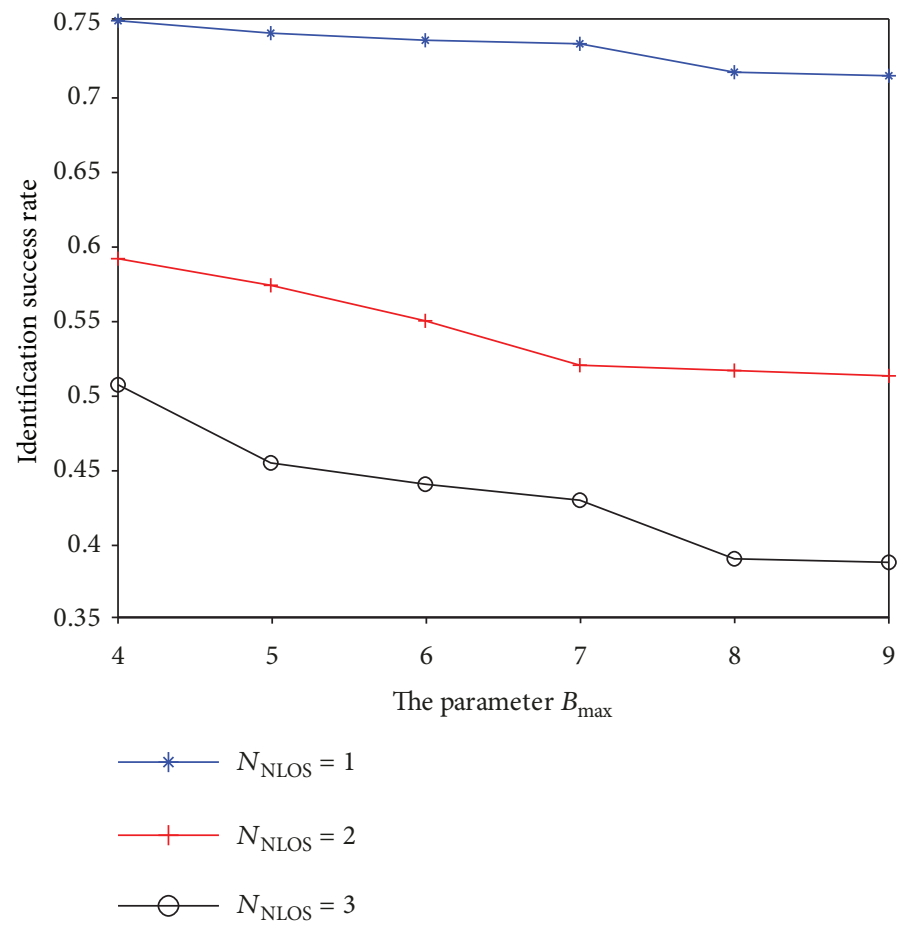

FIgURE 3: The identification success rate versus $B_{\max }$.

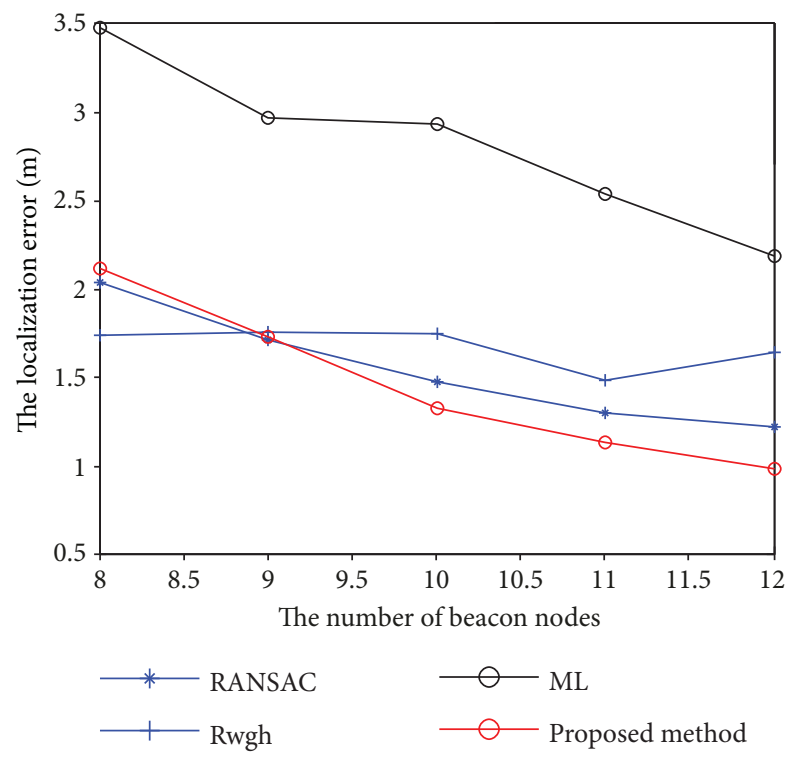

Figure 4: The localization error versus the number of beacon nodes.

of the measurement noise in the LOS condition $\sigma_{i}$ is 1 . The results show that when the number of measurements in the NLOS condition is equal, the success rate is less affected by $B_{\max }$. However, as the number of measurements in the NLOS condition increases, the success rate of the proposed method decreases.

In Figure 4 we evaluate the impact of the number of beacon nodes on the localization error. The results show that the localization error decreases as the number of beacon nodes increases. In addition, the ML method has the largest

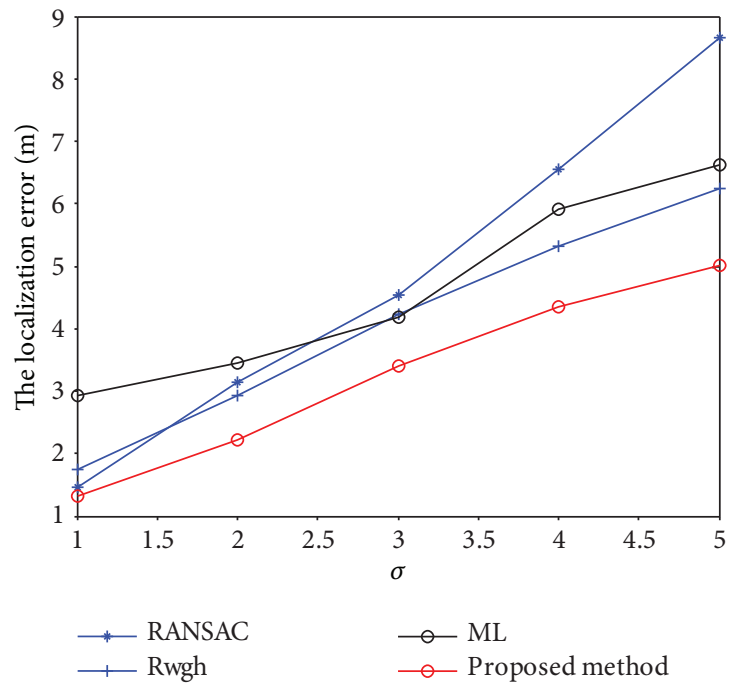

FIgURE 5: The localization error versus $\sigma_{i}$.

localization error. When the number of beacon nodes is relatively fewer, such as 8 , the Rwgh method works best. When the number of beacon nodes is 9 , the localization errors of the Rwgh method, the RANSAC method, and the proposed method are approximately the same. However, the localization error of the proposed method declines faster, and this method has the highest localization accuracy when the number of beacon nodes increases.

Figure 5 shows the relation between the localization error and the standard variance of the measurement noise $\sigma_{i} . \sigma_{i}$ is varied from 1 to 5 . The results show that as the value of $\sigma_{i}$ 


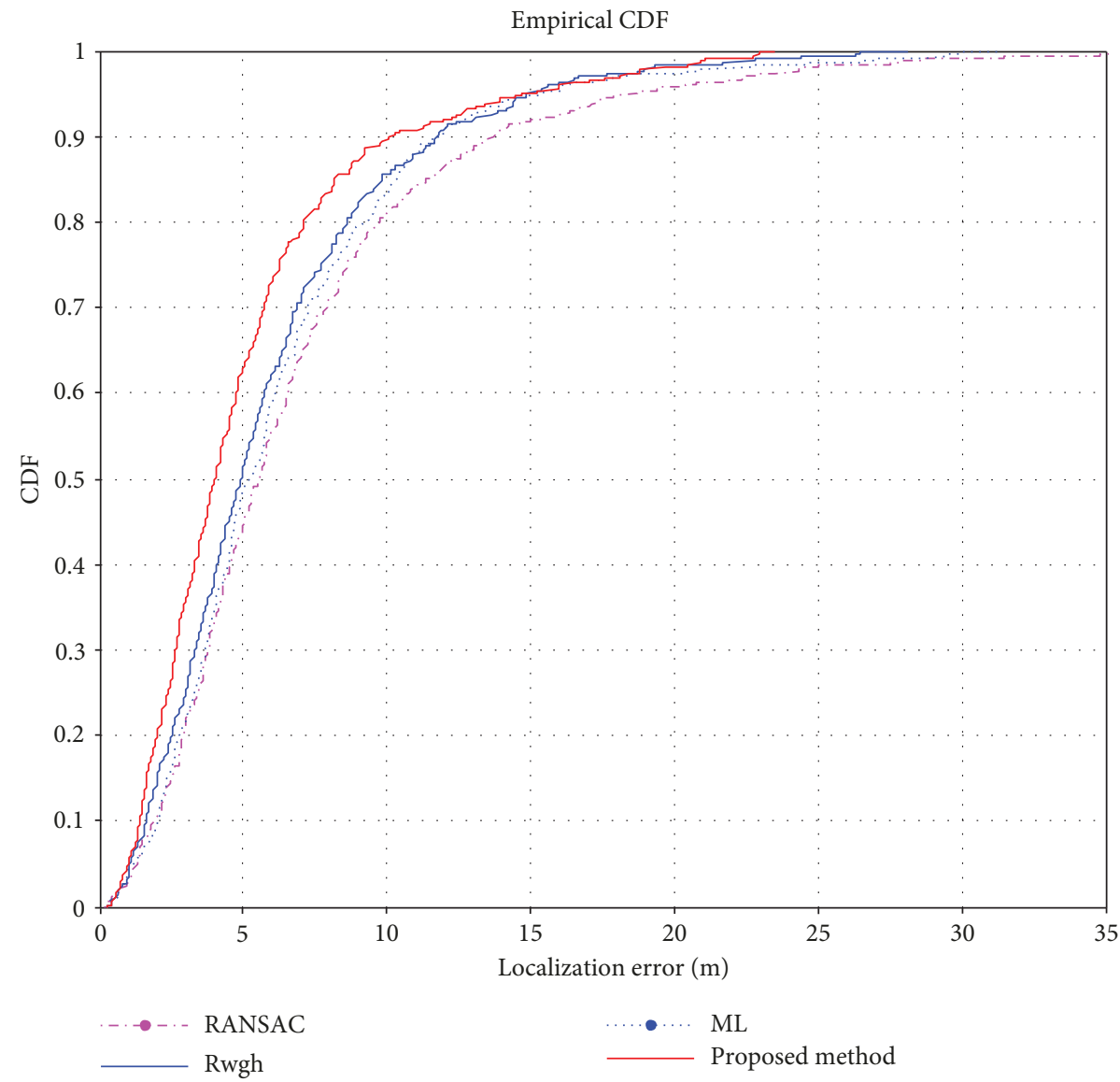

FIgure 6: The CDF of localization error.

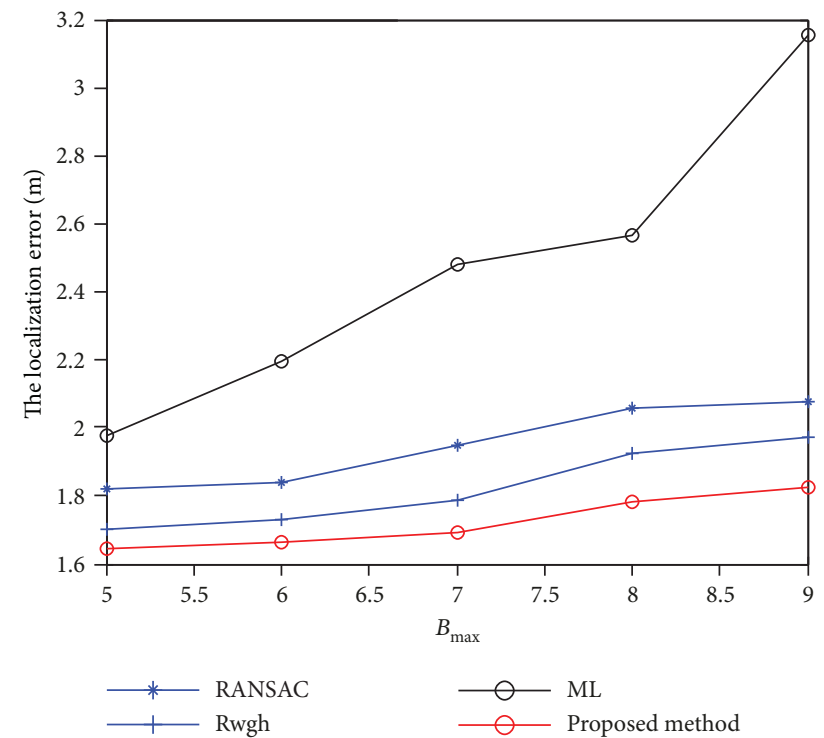

FIgURE 7: The localization error versus $B_{\max }$.

increases, the localization error increases. In addition, the proposed method has the highest localization accuracy compared with the other methods. In comparison with ML, RANSAC, and Rwgh methods, the localization accuracy of

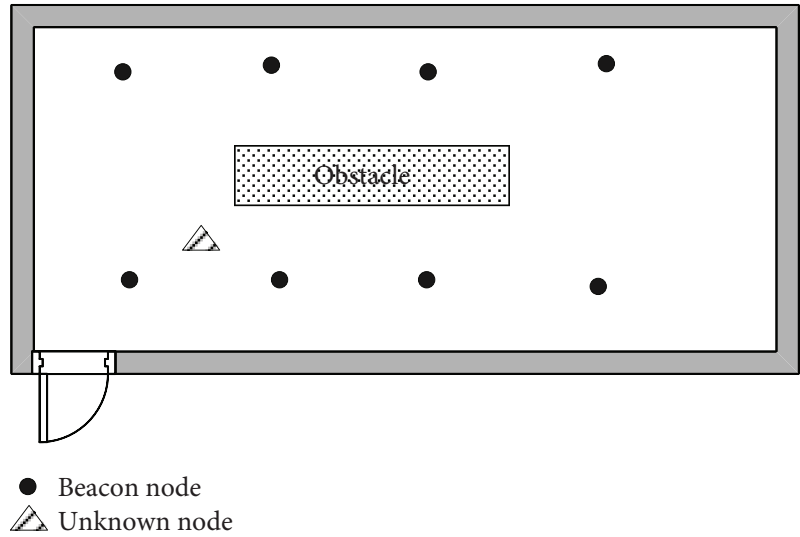

Figure 8: The floor plan for the test bed.

the proposed method increases to $29.36 \%, 33.05 \%$, and $20.37 \%$, respectively.

Figure 6 shows the CDF of the localization error when the NLOS error obeys the uniform distribution $n_{\mathrm{NLOS}} \sim U(0,6)$. We can see that the $80 \%$ localization error of the proposed method is less than $7.122 \mathrm{~m}$, and the CDF trends toward one with a localization error of less than $22.7 \mathrm{~m}$. In comparison with the $80 \%$ localization error of the Rwgh, ML, and RANSAC methods, $7.2 \mathrm{~m}, 8.1 \mathrm{~m}$, and $6.1 \mathrm{~m}$ are achieved, respectively. 


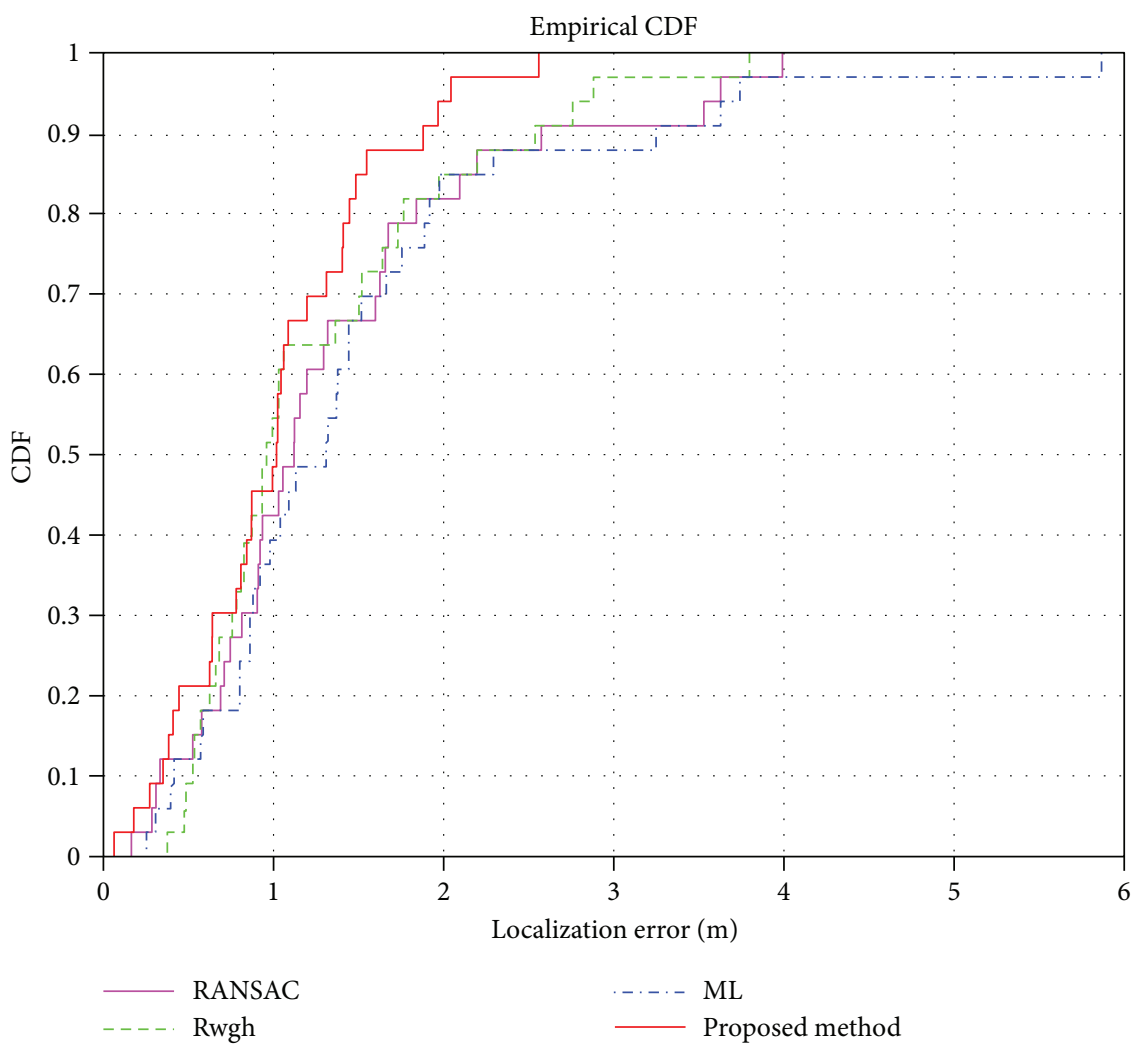

FIgURE 9: The CDF of localization error in realistic experiment.

Figure 7 shows the impact of the parameter $B_{\max }$ on the localization error. As the value of $B_{\max }$ increases, the localization errors of all algorithms are increasing. However, the ML method is seriously affected by $B_{\max }$. The proposed method achieves the lowest localization error. In comparison with ML, RANSAC, and Rwgh methods, the localization accuracy of the proposed method increases to $30.46 \%, 11.68 \%$, and $5.64 \%$, respectively.

In order to verify the effective of the proposed localization method, we perform the realistic experiment in the indoor environment. As shown in Figure 8, eight beacon nodes and one unknown node are deployed in the room. The beacon node and unknown node are installed up to $1.7 \mathrm{~m}$ above the ground. The experimental equipment is chirp spread spectrum (CSS) localization system.

Figure 9 shows the CDF of the localization error in realistic experiment. It can be seen that $80 \%$ localization error of the proposed method is less than $1.879 \mathrm{~m}$. The CDF trends toward one with a localization error of less than $2.559 \mathrm{~m}$. The average localization error of the proposed method is $1.0205 \mathrm{~m}$. The average localization errors of RANSAC, Rwgh, and ML are $1.3491 \mathrm{~m}, 1.2603 \mathrm{~m}$, and $1.5036 \mathrm{~m}$, respectively.

\section{Conclusion}

The NLOS problem is one of the most challenging problems for wireless sensor networks. It can seriously reduce localization accuracy. In this paper, the TOA measurement model is first introduced. We then proposed an NLOS identification method based on residual analysis to solve the problem caused by the NLOS error. In addition, the particle swarm optimization with a constriction factor algorithm is proposed to find the optimal solution of the location estimate of an unknown node. Simulation results show that this method can reduce the influence of NLOS error and improve the positioning accuracy, especially when the number of beacon nodes is relatively large. In future work, the proposed method could be extended to the distributed localization method. At the same time, we will modify the residual analysis method and apply it to the mobile localization to improve the effectiveness of particle filter.

\section{Appendix}

In this section, we introduce the maximum likelihood localization method. We assume that the position of the beacon node is denoted as $\left[\left(x_{1}, y_{1}\right), \ldots,\left(x_{N}, y_{N}\right)\right]$. The position of an unknown node is $\boldsymbol{\theta}=\left[x_{u}, y_{u}\right]^{\mathrm{T}} . \widehat{d}_{i}$ is the measurement distance for the $i$ th beacon node.

$$
\begin{gathered}
\left(x_{1}-x_{u}\right)^{2}+\left(y_{1}-y_{u}\right)^{2}=\left(\widehat{d}_{1}\right)^{2}, \\
\vdots \\
\left(x_{N}-x_{u}\right)^{2}+\left(y_{N}-y_{u}\right)^{2}=\left(\widehat{d}_{N}\right)^{2} .
\end{gathered}
$$


The above equation can be represented by a linear equation $\mathbf{A} \cdot \boldsymbol{\theta}=\mathbf{B}$, where $\mathbf{A}$ and $\mathbf{B}$ are given by

$$
\begin{aligned}
\mathbf{A} & =2\left[\begin{array}{cc}
\left(x_{1}-x_{2}\right) & \left(y_{1}-y_{2}\right) \\
\left(x_{1}-x_{3}\right) & \left(y_{1}-y_{3}\right) \\
\vdots & \vdots \\
\left(x_{1}-x_{N}\right) & \left(y_{1}-y_{N}\right)
\end{array}\right], \\
\mathbf{B} & =\left[\begin{array}{c}
\left(\hat{d}_{2}\right)^{2}-\left(\hat{d}_{1}\right)^{2}-\left(x_{2}^{2}+y_{2}^{2}\right)+\left(x_{1}^{2}+y_{1}^{2}\right) \\
\left(\hat{d}_{3}\right)^{2}-\left(\hat{d}_{1}\right)^{2}-\left(x_{3}^{2}+y_{3}^{2}\right)+\left(x_{1}^{2}+y_{1}^{2}\right) \\
\vdots \\
\left(\hat{d}_{N}\right)^{2}-\left(\hat{d}_{1}\right)^{2}-\left(x_{N}^{2}+y_{N}^{2}\right)+\left(x_{1}^{2}+y_{1}^{2}\right)
\end{array}\right] .
\end{aligned}
$$

We can obtain the estimated position of the unknown node as follows:

$$
\widehat{\boldsymbol{\theta}}=\left(\mathbf{A}^{\mathrm{T}} \mathbf{A}\right)^{-1} \mathbf{A}^{\mathrm{T}} \mathbf{B}
$$

\section{Data Availability}

The data used to support the findings of this study are available from the corresponding author upon request.

\section{Conflicts of Interest}

The authors declare that there is no conflict of interest regarding the publication of this paper

\section{Acknowledgments}

This work was supported by the National Natural Science Foundation of China under Grant no. 61803077, the Natural Science Foundation of Hebei Province under Grant no. F2016501080, and the Fundamental Research Funds for the Central Universities of China under Grant nos. N172304024 and N152302001.

\section{References}

[1] P. H. Tsai, R. G. Tsai, and S. S. Wang, "Hybrid localization approach for underwater sensor networks," Journal of Sensors, vol. 2017, Article ID 5768651, 13 pages, 2017.

[2] S. Xie and Y. Wang, "Construction of tree network with limited delivery latency in homogeneous wireless sensor networks," Wireless Personal Communications, vol. 78, no. 1, pp. 231-246, 2014.

[3] S. Subedi and J. Y. Pyun, "Practical fingerprinting localization for indoor positioning system by using beacons," Journal of Sensors, vol. 2017, Article ID 9742170, 16 pages, 2017.

[4] Y. Ahmadi, N. Neda, and R. Ghazizadeh, "Range free localization in wireless sensor networks for homogeneous and nonhomogeneous environment," IEEE Sensors Journal, vol. 16, no. 22, pp. 8018-8026, 2016.

[5] K. Subbu, C. Zhang, J. Luo, and A. Vasilakos, "Analysis and status quo of smartphone-based indoor localization systems," IEEE Wireless Communications, vol. 21, no. 4, pp. 106-112, 2014.

[6] Y. T. Chan, W. Y. Tsui, H. C. So, and P. C. Ching, "Time-ofarrival based localization under NLOS conditions," IEEE Transactions on Vehicular Technology, vol. 55, no. 1, pp. 17-24, 2006.

[7] J. Cota-Ruiz, P. Rivas-Perea, E. Sifuentes, and R. GonzalezLandaeta, "A recursive shortest path routing algorithm with application for wireless sensor network localization," IEEE Sensors Journal, vol. 16, no. 11, pp. 4631-4637, 2016.

[8] W. Liu, D. Wang, H. Jiang, W. Liu, and C. Wang, "An approximate convex decomposition protocol for wireless sensor network localization in arbitrary-shaped fields," IEEE Transactions on Parallel and Distributed Systems, vol. 26, no. 12, pp. 3264-3274, 2015.

[9] Z. Yang, C. Wu, T. Chen, Y. Zhao, W. Gong, and Y. Liu, "Detecting outlier measurements based on graph rigidity for wireless sensor network localization," IEEE Transactions on Vehicular Technology, vol. 62, no. 1, pp. 374-383, 2013.

[10] C. Müller, D. I. Alves, B. F. Uchôa-Filho, R. Machado, J. B. S. Martins, and L. L. de Oliveira, "Improved solution for node location multilateration algorithms in wireless sensor networks," Electronics Letters, vol. 52, no. 13, pp. 1179-1181, 2016.

[11] P. C. Chen, "A non-line-of-sight error mitigation algorithm in location estimation," in Proceedings of thewireless communication and networking conference, pp. 316-320, New Orleans, LA, USA, 1999.

[12] I. Rasool and A. H. Kemp, "Statistical analysis of wireless sensor network Gaussian range estimation errors," IET Wireless Sensor Systems, vol. 3, no. 1, pp. 57-68, 2013.

[13] W. Li, Y. Jia, J. Du, and J. Zhang, "Distributed multiple-model estimation for simultaneous localization and tracking with NLOS mitigation," IEEE Transactions on Vehicular Technology, vol. 62, no. 6, pp. 2824-2830, 2013.

[14] R. Vaghefi, J. Schloemann, and R. Buehrer, "NLOS mitigation in TOA-based localization using semidefinite programming," in 2013 10th Workshop on Positioning, Navigation and Communication (WPNC), pp. 1-6, Dresden, Germany, March 2013.

[15] U. Hammes and A. M. Zoubir, "Robust mobile terminal tracking in NLOS environments based on data association," IEEE Transactions on Signal Processing, vol. 58, no. 11, pp. 58725882, 2010.

[16] L. Cheng, C.-D. Wu, Y.-Z. Zhang, and Y. Wang, "An indoor localization strategy for a mini-UAV in the presence of obstacles," International Journal of Advanced Robotic Systems, vol. 9, no. 4, p. 153, 2017.

[17] X. Yu, C. Wu, and L. Cheng, "Indoor localization algorithm for TDOA measurement in NLOS environments," IEICE Transactions on Fundamentals of Electronics, Communications and Computer Sciences, vol. E97.A, no. 5, pp. 1149-1152, 2014.

[18] X. Wang, L. Ding, and S. Wang, "Trust evaluation sensing for wireless sensor networks," IEEE Transactions on Instrumentation and Measurement, vol. 60, no. 6, pp. 2088-2095, 2011.

[19] C. Yang, B. Chen, and F. Liao, "Mobile location estimation using fuzzy-based IMM and data fusion," IEEE Transactions on Mobile Computing, vol. 9, no. 10, pp. 1424-1436, 2010.

[20] L. Cheng, Y. Wang, H. Wu, N. Hu, and C. Wu, "Non-parametric location estimation in rough wireless environments for 
wireless sensor network," Sensors and Actuators A: Physical, vol. 224, pp. 57-64, 2015.

[21] Y. Wang, L. Cheng, and N. Hu, "Bayes sequential test based NLOS localization method for wireless sensor network," in The 27th Chinese Control and Decision Conference (2015 CCDC), pp. 5230-5234, Qingdao, China, May 2015.

[22] R. M. Vaghefi and R. M. Buehrer, "Cooperative sensor localization with NLOS mitigation using semidefinite programming," in 2012 9th Workshop on Positioning, Navigation and Communication, pp. 13-18, Dresden, Germany, March 2012. 


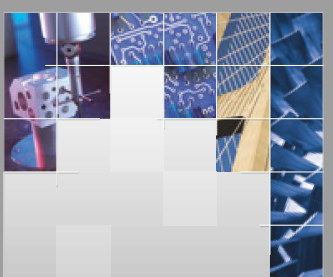

\section{Enfincering}
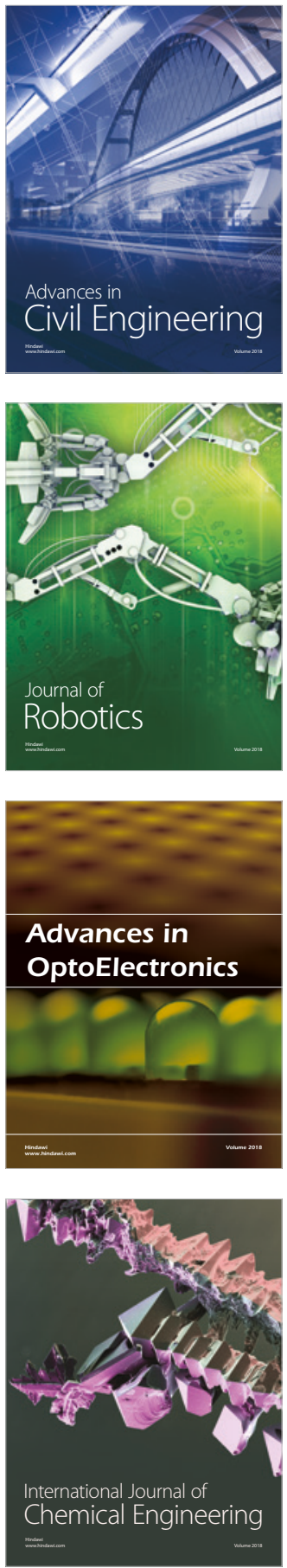

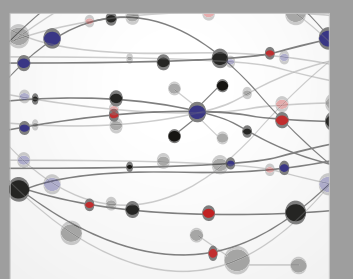

\section{Rotating \\ Machinery}

The Scientific World Journal

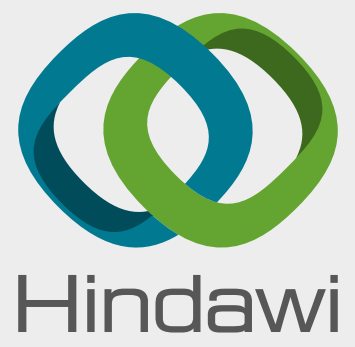

Submit your manuscripts at

www.hindawi.com
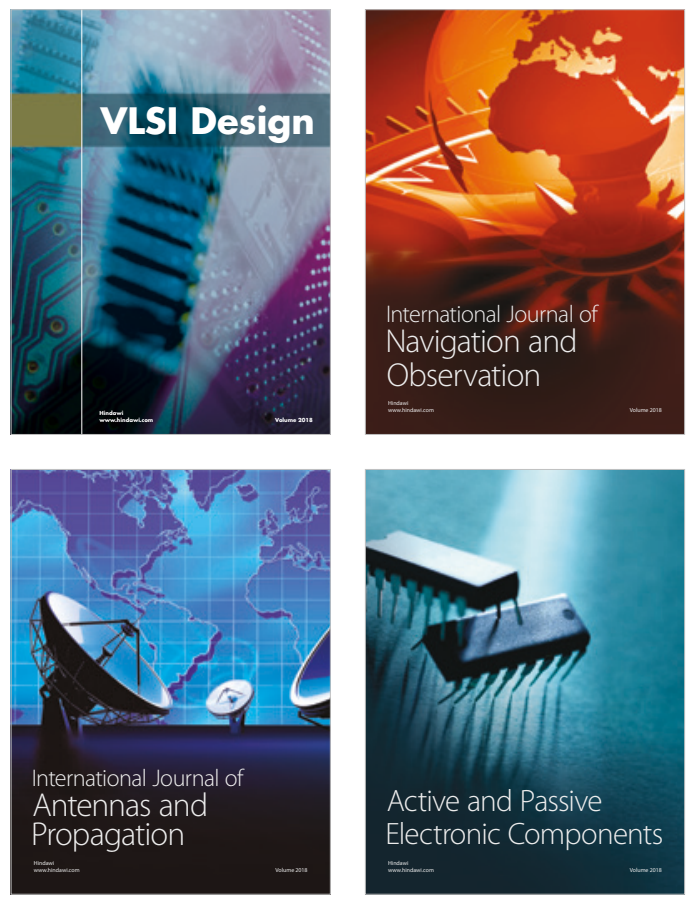
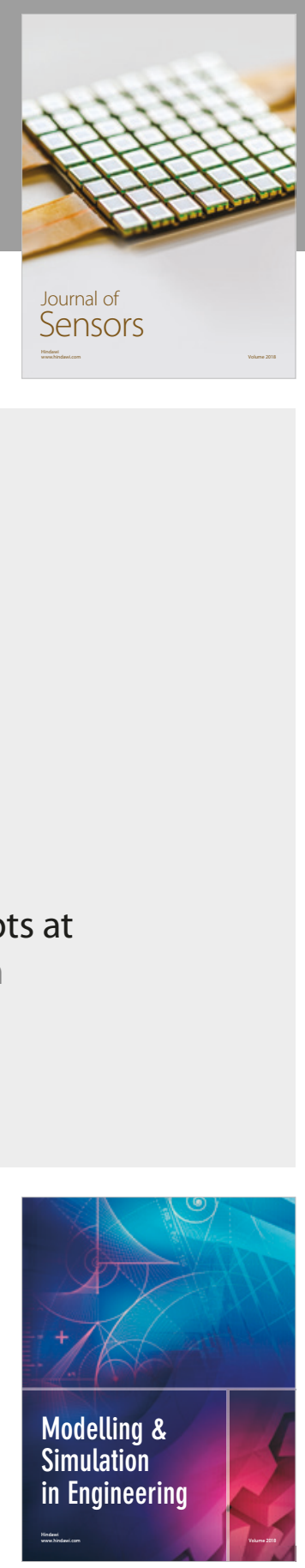

\section{Advances \\ Multimedia}
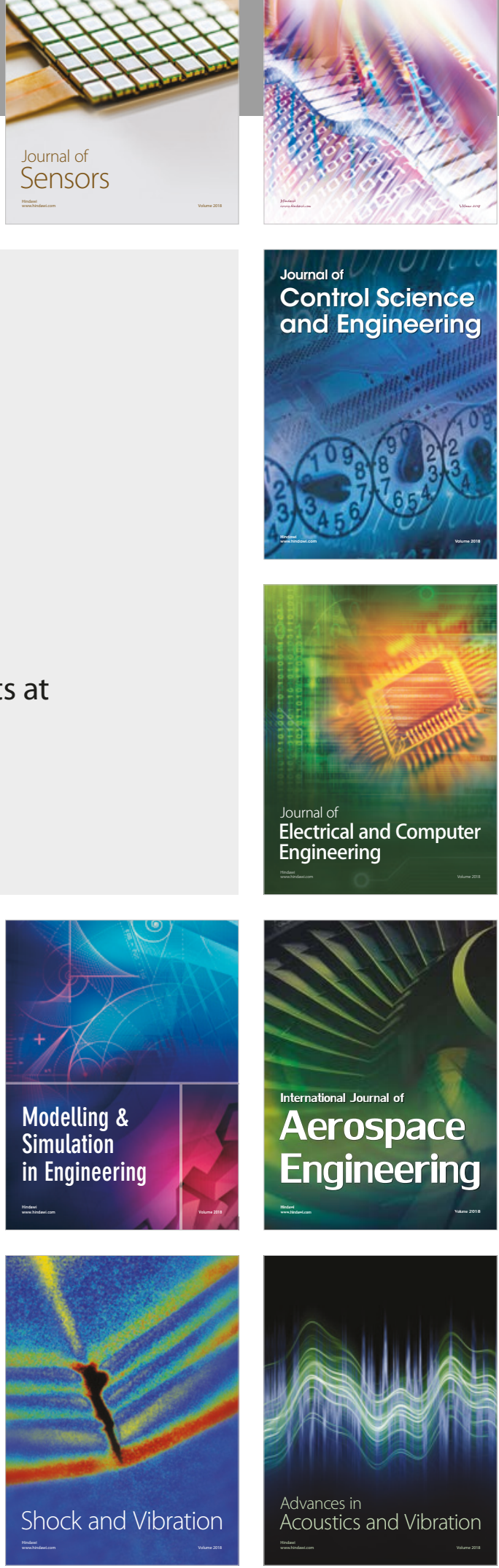